SAND $2000-2379 \mathrm{C}$

\title{
Uncertainty Estimation in the Determination of Thermal Conductivity of 304 Stainless Steel ${ }^{1}$
}

\author{
Bennie F. Blackwell ${ }^{2}$, Walter Gill ${ }^{3}$, Kevin J. Dowding ${ }^{4}$ and Robert G. Easterling ${ }^{5}$ \\ Sandia National Laboratories \\ MS 0828 \\ Albuquerque, NM 87185
}

\begin{abstract}
The thermal conductivity of 304 stainless steel has been estimated from transient temperature measurements and knowing the volumetric heat capacity. Sensitivity coefficients were used to guide the design of this experiment as well as to estimate the confidence interval in the estimated thermal conductivity. The uncertainty on the temperature measurements was estimated by several means, and its impact on the estimated conductivity is discussed. The estimated thermal conductivity of 304 stainless steel is consistent with results from other sources.
\end{abstract}

\section{NOMENCLATURE}

c specific heat, J/Kg-K

$k$ thermal conductivity, $\mathrm{W} / \mathrm{m}-\mathrm{K}$

$N_{p} \quad$ number of parameters

$N_{s} \quad$ number of sensors

$N_{t} \quad$ number of measurement times

$S \quad$ sum of squares function, see Eq. (1)

$T \quad$ temperature, ${ }^{\circ} \mathrm{C}$

$T_{k} \quad$ scaled sensitivity coefficient, ${ }^{\circ} \mathrm{C}$, see Eq. (4)

$T_{\max }$ maximum temperature, ${ }^{\circ} \mathrm{C}$

$T_{\min } \quad$ minimum temperature, ${ }^{\circ} \mathrm{C}$

$t \quad$ time, $s$

$X \quad$ sensitivity matrix

1. Sandia is a multiprogram laboratory operated by Sandia Corporation, a Lockheed Martin Company, for the United States Department of Energy under Contract DE-AC04-94AL85000.

2. Distinguished Member of Technical Staff, Fellow ASME.

3. Principal Member of Technical Staff, Member ASME.

4. Senior Member of Technical Staff, Member ASME.

5. Senior Scientist.

\author{
$\vec{x} \quad$ position vector \\ $Y_{i j} \quad$ temperature measurement for sensor $i$ at time $j,{ }^{\circ} \mathrm{C}$ \\ Greek \\ $\Delta \quad$ D-optimality condition, see Eq. (8) \\ $\Delta^{+} \quad$ dimensionless D-optimality condition, see Eq. (9) \\ $\Delta T \quad=T_{\max }-T_{\min }$ \\ $\Delta t \quad$ data sample rate \\ $\alpha \quad$ thermal diffusivity, $\mathrm{m}^{2} / \mathrm{s}$ \\ $\rho$ density, $\mathrm{Kg} / \mathrm{m}^{3}$ \\ $\hat{\sigma}_{p} \quad$ estimated standard deviation in parameter $p$, units of $p$ \\ $v \quad$ iteration counter

\section{INTRODUCTION}

Computational techniques for conduction heat transfer have evolved to the point where the more significant numerical issues have been resolved. There are any number of numerical algorithms that can be used to accurately compute temperature fields; these include finite difference, finite volume, and finite element. The focus is beginning to turn toward validation of these computational models. In the context of this paper, we adopt the definition of Roache (1998) that validation is "solving the right set of equations" as opposed to verification which is "solving the set of equations right." Validation of CFD models is discussed in Stern et al. (1999) and the references contained therein. Our work is ultimately directed toward the validation of thermal models of complex systems.

In order to make a quantitative statement about the level of validation of a simulation, one must estimate the uncertainty in both the simulations and the experiment. The experimental 


\section{DISCLAIMER}

This report was prepared as an account of work sponsored by an agency of the United States Government. Neither the United States Government nor any agency thereof, nor any of their employees, make any warranty, express or implied, or assumes any legal liability or responsibility for the accuracy, completeness, or usefulness of any information, apparatus, product, or process disclosed, or represents that its use would not infringe privately owned rights. Reference herein to any specific commercial product, process, or service by trade name, trademark, manufacturer, or otherwise does not necessarily constitute or imply its endorsement, recommendation, or favoring by the United States Government or any agency thereof. The views and opinions of authors expressed herein do not necessarily state or reflect those of the United States Government or any agency thereof. 


\section{DISCLAIMER}

Portions of this document may be illegible in electronic image products. Images are produced from the best available original document. 
community is very familiar with the root-sum-of-squares technique for propagation of experimental uncertainty; see Coleman and Steele (1999) for a discussion of this methodology. This same methodology can be used to propagate uncertainty through a computational model; the computational community is not as progressed as the experimental community in applying these techniques. In the extension of the root sumof-squares methodology to computational models, sensitivity coefficients of the various model parameters (material properties, etc.) must be known, along with their corresponding uncertainty, in order to perform an uncertainty analysis. In this work sensitivity coefficients are defined as partial derivatives of field variables (temperature in this case) with respect to model parameters (thermal conductivity in this case); see Blackwell et al. (1999) for a discussion of numerical techniques for computing sensitivity coefficients in thermal problems. This work focuses on estimating the thermal conductivity of 304 stainless steel and estimating the associated experimental uncertainty and should be viewed as one of the first steps in validating a computational model.

As an aside, the process of uncertainty estimation (both experimental and computational) should not be viewed as an attempt to quantify uncertainty that is accurate to $n$ significant digits (where $n$ is known). Instead, a significant (and often primary) benefit of the uncertainty analysis is to identify those parameters that dominate the overall uncertainty and then take appropriate steps to reduce the overall uncertainty to a programatically acceptable ${ }^{1}$ level. The uncertainty analysis often leads to a road map on how to best spend project resources; focus on those important parameters and be satisfied with nominal or handbook values for those unimportant parameters. A quotation by Dowdell (1982) summarizes these sentiments: "There is a lot of uncertainty on uncertainty analysis but some analysis is better than no analysis at all."

\section{Description of Experimental Hardware}

A cutaway view of the experiment is shown in Fig. 1; additional details have been presented in Blackwell et al. (2000). The hollow stainless steel cylinder has an outside diameter of $8.89 \mathrm{~cm}(3.5 \mathrm{in})$, wall thickness of $0.508 \mathrm{~cm}(0.2$ in), and length of $13.97 \mathrm{~cm}(5.5 \mathrm{in})$. A flange of $12.7 \mathrm{~cm}(5.0 \mathrm{in})$ diameter by $0.635 \mathrm{~cm}(0.25 \mathrm{in})$ thickness is present on the upper and lower cylinder ends. The top and bottom copper blocks are each composed of two halves: the (solid) contact plate is 12.7 $\mathrm{cm}(5.0 \mathrm{in})$ diameter by $1.905 \mathrm{~cm}(0.75 \mathrm{in})$ thick; the body is $12.7 \mathrm{~cm}(5.0 \mathrm{in})$ diameter by $3.848 \mathrm{~cm}(1.515 \mathrm{in})$ and has serpentine channels machined in it to enhance the heat exchange effectiveness for the fluid circulated through it. The contact plate and body are brazed together and are referred to collectively as the copper block.

1. Each program or project will have its objectives and budget. The integration of objectives, budget, etc., will define programmatically acceptable level.
Time dependent temperature measurements are provided by thermocouples mounted in the top and bottom copper blocks identified in Fig. 1. A single 30-gauge thermocouple $(0.254$ $\mathrm{mm}, 0.010$ in diameter, Type $\mathrm{K}$ ) measures the temperature of each of the copper blocks; this thermocouple is located 4.483 $\mathrm{cm}$ (1.765 in) from the copper/stainless steel flange interface (in the body) and at the bottom of a $6.35 \mathrm{~cm}$ ( 2.25 in) deep radial hole. Two separate temperature controlled baths supply fluid to the top/bottom (Oxygen Free High Conductivity, OFHC) copper blocks. A simplified cross section of the heating/cooling blocks and stainless steel cylinder is shown in Fig. 2.

Thermocouples are mounted in the stainless steel cylinder walls at fourteen axial stations with a uniform spacing of 0.953 $\mathrm{cm}(0.375 \mathrm{in})$. Station 1 is near the top of the cylinder and is located $0.795 \mathrm{~cm}(0.313 \mathrm{in})$ from the inside face of the flange. Station 14 is located at a mirror image position near the bottom flange. Stations 7 and 8 are located at $\pm 0.475 \mathrm{~cm}( \pm 0.187$ in) from the $x=0$ position (axial midplane), respectively. At each axial station along the stainless steel cylinder there are thermocouples at four angular stations, each 90 degrees apart for a total of 56 thermocouples mounted in the cylinder wall.

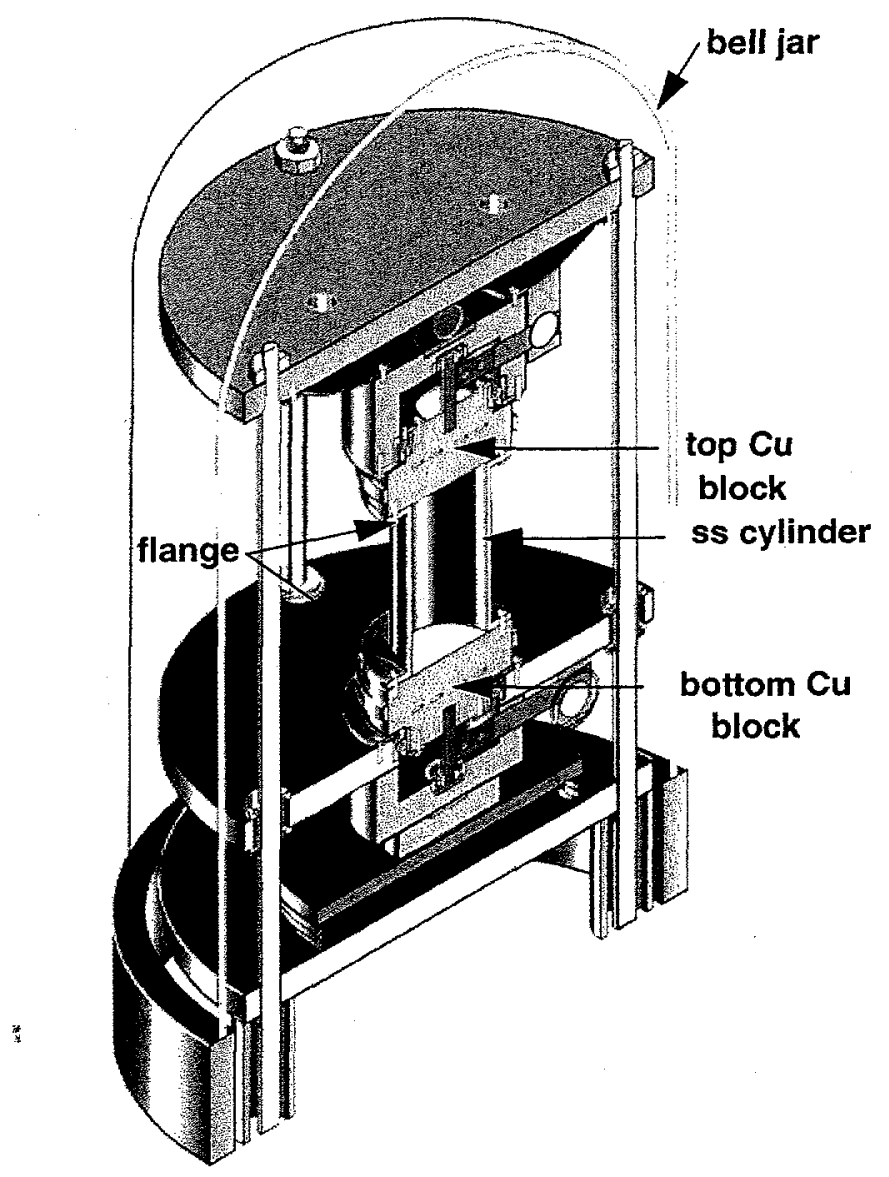

Figure 1. Cutaway view of experiment

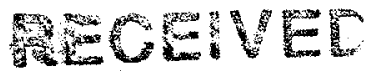




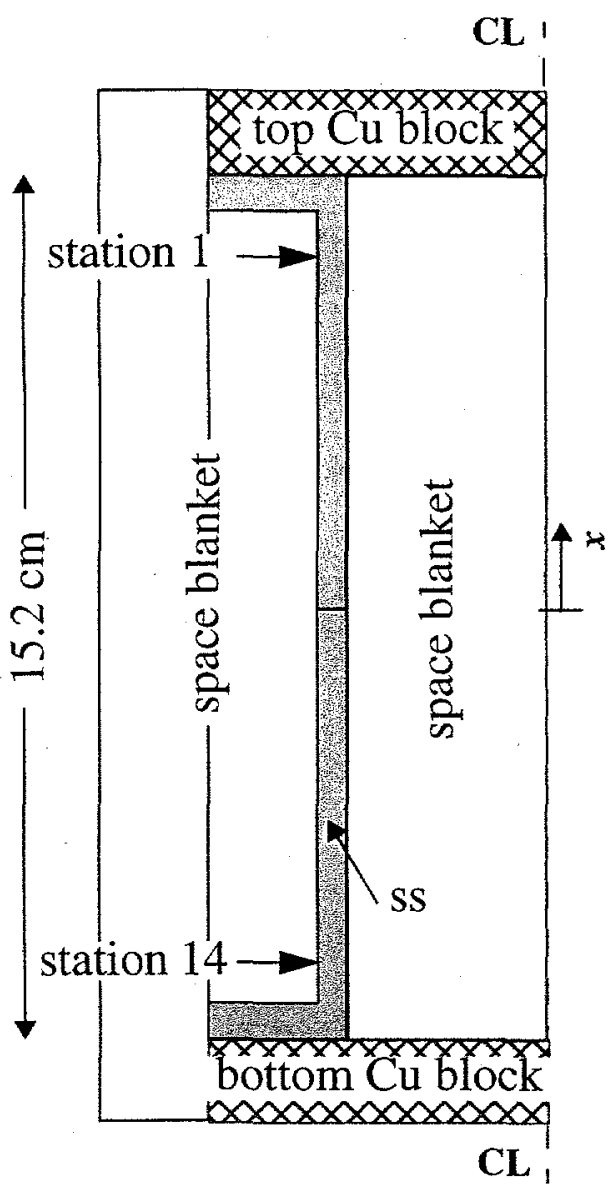

Figure 2. Cross section of experimental apparatus

The thermocouples are arranged in four columns: two columns ( $A$ and $C$ ) of Type $K$ and two columns (B and D) of Type T, both of 30-gage wire $(0.254 \mathrm{~mm}$ or 0.010 in diameter $)$.

The thermocouples were installed in a thermowell by drilling $1.17 \mathrm{~mm}$ (0.0461 in) diameter by $2.54 \mathrm{~mm}(0.1 \mathrm{in})$ deep holes in the cylinder walls. Each thermocouple was formed by stripping insulation from single strand wire, welding the junction, and dipping the junction in a eutectic mix of indium/tin. The eutectic was also placed in the thermowells, and it remains liquid and ensures good contact between the thermoelectric elements and the stainless steel cylinder. The leads were wrapped around the outside diameter of the cylinder for $1 / 8$ th turn and held down with Kapton ${ }^{\mathrm{TM}}$ tape so as to minimize conduction losses along the lead wires.

A hollow cylinder was chosen because the hardware is also used for a companion contact conductance experiment. Some details of this experiment are given in Blackwell et al. (2000). Two-dimensional axisymmetric simulations of the cross section in Fig. 2 have demonstrated that the temperature profile in the cross sections at Stations 1 and 14 is uniform. Consequently, over the instrumented portion of the cylinder, heat conduction can be considered one dimensional.

The vacuum system consists of a bell jar vacuum chamber, a base plate with feed-through ports, and a complete vacuum pumping system with controls and gaging. The nominal dimensions of the glass bell jar vacuum chamber are $45.7 \mathrm{~cm}$ by $76.2 \mathrm{~cm}$ by $0.826 \mathrm{~cm}$ ( 18 in by 30 in by 0.325 in) wall thickness. The system is composed of a high-speed roughing pump and a four-stage diffusion pump capable of maintaining $10^{-7}$ Torr.

The data was acquired using the PC-based LabView ${ }^{\mathrm{TM}}$ software with a 16 bit A-D system; the sample interval was approximately 1.5 seconds.

\section{EXPERIMENTAL TEMPERATURE RESULTS}

The thermocouple results for a pressure of roughly $4 \times 10^{-5}$ Torr are shown in Fig. 3. Because of the symmetric placement

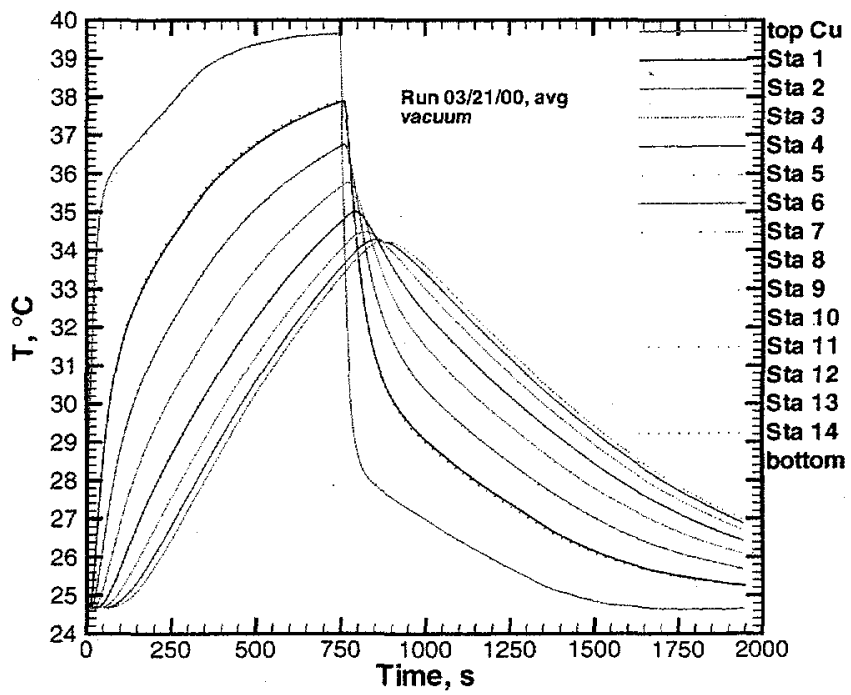

\section{Figure 3. Experimental temperature results for Run 032100.}

of the thermocouples about the axial midplane of the cylinder and the nominally symmetric boundary conditions on the two ends, the experimental results should overlay as pairs of thermocouples. The maximum difference in the top and bottom copper block temperatures is of the order of $0.1^{\circ} \mathrm{C}$ or less, indicating very good end-to-end symmetry. The remaining seven pairs $(1: 14,2: 13$, etc. $)$ of thermocouples exhibit even better symmetry. The results presented here are better than the results presented in Blackwell et al. (2000) for three reasons. First, larger capacity fluid baths were used, and consequently, the temperature rise rate of the top and bottom copper blocks is greater; this will produce larger thermal conductivity sensitivity coefficients. Second, the fluid delivery system to the top and bottom copper blocks was modified with the result being significantly improved end-to-end symmetry of the temperature profiles. Third, the thermocouple lead wire wrap was reduced from 1 turn to $1 / 8$ th turn; this reduces the thermal mass not 
accounted for in the model without introducing axial conduction errors along the lead wires. While the fluid baths temperature was measured, the results are not presented here since they were not used in the data reduction procedure.

Standard emf-temperature calibration curves were used in the data reduction process. In an attempt to remove bias, approximately $39 \mathrm{~s}$ of thermocouple data (sampled at approximately $1.5 \mathrm{~s}$ ) was taken for which all thermocouples should be at nominally the same temperature. This is termed a data leader. The data leader was averaged and this result is termed "global average initial temperature." Next, each individual thermocouple was averaged over this same $39 \mathrm{~s}$ interval and this result is termed "local average initial temperature." The difference between the global and local average initial temperature was applied to each individual thermocouple as a bias correction for all measurement times. These bias corrections for each of the $56 \mathrm{~T} / \mathrm{C}$ 's are shown in

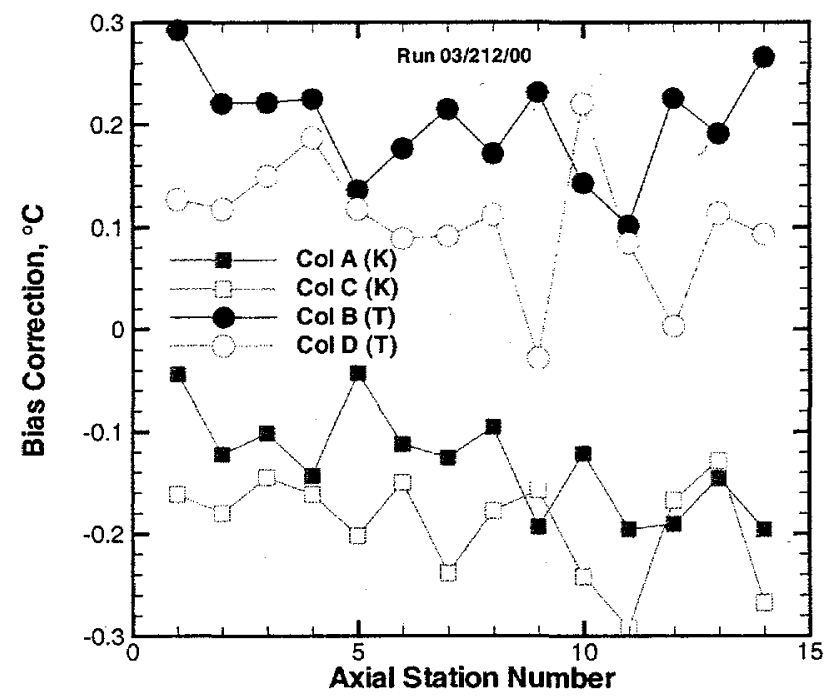

Figure 4. Bias correction for each of $56 \mathrm{~T} / \mathrm{C}$ 's, as determined from the data leader.

Figure 4; Columns $\mathrm{A}$ and $\mathrm{C}$ are Type $\mathrm{K}$ and are consistently lower than the global average whereas Columns $B$ and D are Type $T$ and are consistently higher. As a result of this bias correction, all thermocouples have the same average (over the data leader) initial temperature.

The standard deviation in indicated temperature during the data leader for a given sensor is an indicator of the best that one could hope to do as there are no temperature gradients during this time interval. These standard deviation results are presented in Fig. 5 for the 56 thermocouples. If all 56 thermocouples are lumped together, the standard deviation in temperature for the data leader was estimated to be $0.0023^{\circ} \mathrm{C}$.

The temperature data at each of the 14 axial stations was an average of 4 circumferential stations. The standard deviation in

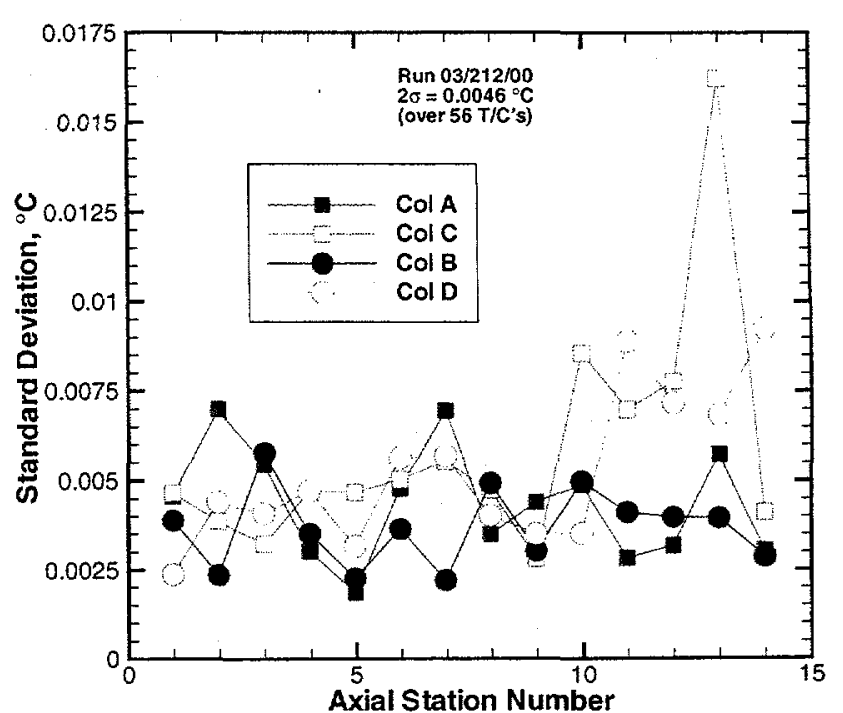

Figure 5. Standard deviation of data leader for each of 56 T/C's.

temperature, based on a sample size of four, was computed for each of the axial stations, and the results are given in Fig. 6.

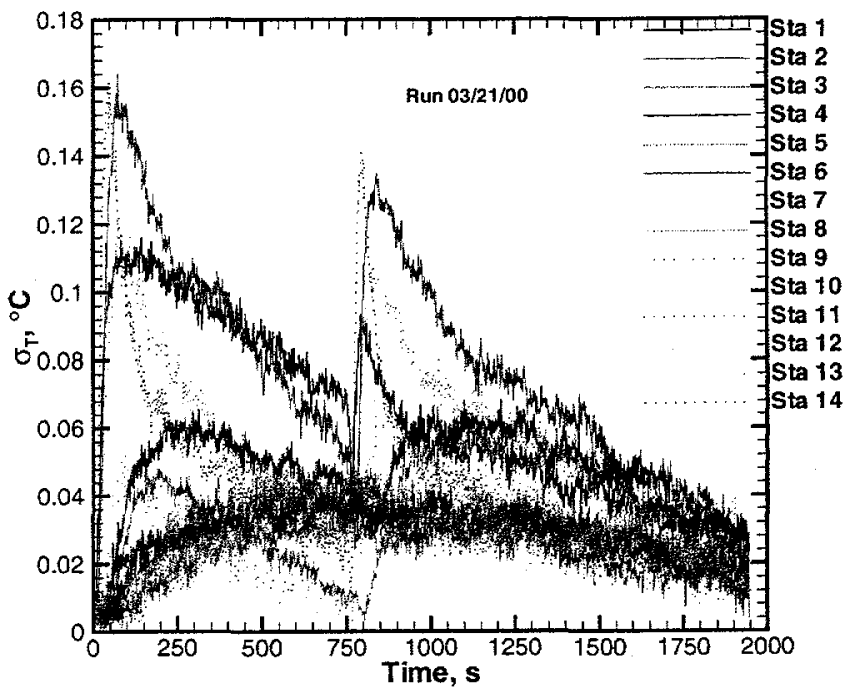

Figure 6. Standard deviation in four circumferential stations for axial Stations 1-14

Stations 1, 2, 13, and 14 exhibit (circumferential) standard deviations that are larger by roughly a factor of two than the remaining 10 stations. The maximum circumferential standard deviation is approximately $0.16^{\circ} \mathrm{C}$. Stations $3-12$ have a standard deviation generally less than $0.06^{\circ} \mathrm{C}$. Since the results in Fig. 6 are taken in the presence of both time and spatial temperature gradients, the standard deviations in Fig. 6 are larger than those in Fig. 5 by an order of magnitude. 
There is a strong correlation between circumferential standard deviation and both temperature rise rate and temperature gradient. Fig. 7 presents the axial temperature gradient $(d T / d x)$, temperature rise rate $(d T / d t)$, and circumferential standard deviation $\left(\sigma_{T}\right)$ for the experimental measurements at Station 14. The temperature rise rate and the temperature gradient for Station 14 were computed using the experimental data and finite differences. The peak spatial temperature gradient lags the peak temperature rise rate. The peak in the standard deviation curve (taken from Fig. 6 for Station 14) occurs at a time between when the maximum time and spatial temperature gradients occur. The maximum temperature gradient at Station 14 is approximately $0.30^{\circ} \mathrm{C} / \mathrm{mm}$. For a thermowell of $1.17 \mathrm{~mm}(0.0461 \mathrm{in})$ diameter, the position of the $0.254 \mathrm{~mm}(0.010 \mathrm{in})$ diameter wire pair could vary by as much as $\pm 0.44 \mathrm{~mm}$ ( $\pm 0.017 \mathrm{in}$ ); this assumes that the two wires are side by side in a direction perpendicular to the long axis of the cylinder. Using the above (maximum) temperature gradient of $0.30^{\circ} \mathrm{C} / \mathrm{mm}$ and the positional uncertainty of the center line of the thermocouple wire, the maximum uncertainty in temperature (due to positional uncertainty) is approximately $\pm 0.13^{\circ} \mathrm{C}$. This result does not consider that the presence of the thermocouple alters the temperature one is trying to measure. The maximum standard deviation in Fig. 6 and the estimated error in temperature because of errors in thermocouple position are consistent.

\section{DESCRIPTION OF THERMOCOUPLE ERRORS}

Thermocouple errors are numerous and have been discussed by a host of others. It is not the intent of this work to review thermocouple errors in general. However, it is appropriate to discuss some of the thermocouple errors that are likely to be

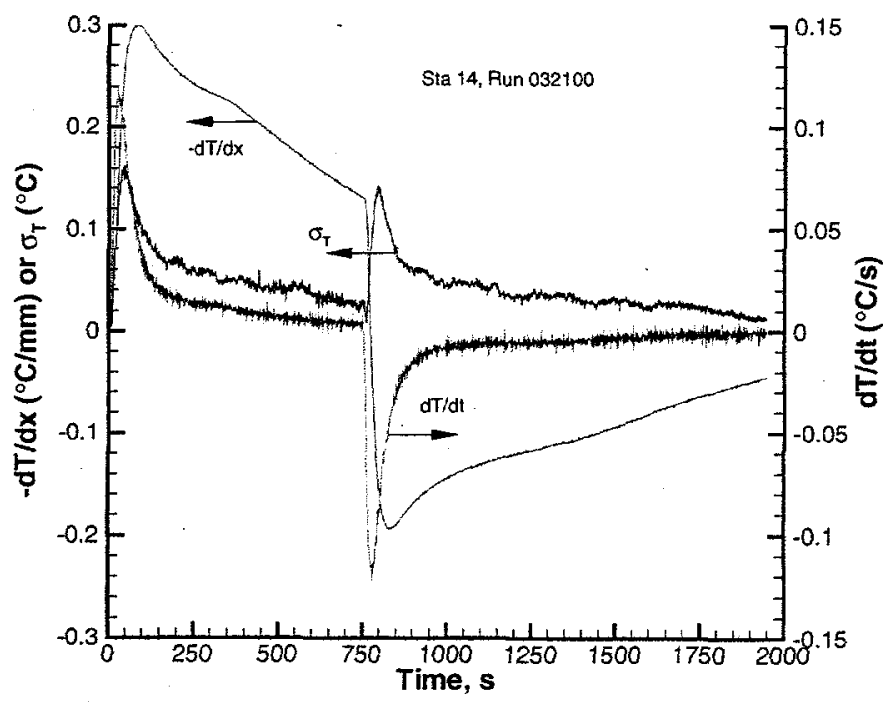

Figure 7. Temperature gradient, temperature rise rate, and circumferential standard deviation for axial station 14, all experimentally based. present in our experiment.

The thermoelectric elements of this experiment sometimes operate in regions of nonuniform temperature. Elementary thermoelectric theory assumes the junction is in a region of uniform temperature. Consequently, the issue of conversion of a voltage to temperature is clouded. These errors will be larger when the axial temperature gradients are large.

Because of the manner in which the thermoelectric elements are installed in the thermowell, there will be a time delay in the thermal (and, hence, electrical) response of the thermocouple. These errors are proportional to the time rate of change of the temperature. During heat up the indicated temperature will be low relative to the undisturbed temperature, while during cool down the indicated temperature will be high.

The location of the thermowell, as well as the position of the thermocouple in the thermowell, are uncertain, with the latter being the larger of the two. The presence of the thermowell will also alter the temperature one is trying to measure.

Deviations of the composition of the actual thermoelectric elements for the thermoelectric elements used in the calibration of this thermocouple type will cause the emf-to-temperature conversion to be in error.

The measurement of the thermocouple emf will have errors, although this is quite small with modern data acquisition systems. The manner in which the reference temperature junction is handled will impact the temperature measurements.

To quantify these errors is difficult and challenging and at best we will only be able to estimate them.

\section{THERMAL CONDUCTIVITY ESTIMATION}

The thermal conductivity is determined by use of nonlinear, least squares parameter estimation techniques; see Beck and Arnold (1977) for additional details. A two-dimensional ${ }^{2}$ computational model of the experiment was developed with thermal conductivity as a parameter. The thermal conductivity is estimated from the experimental temperatures in Fig. 3 such that the least-squares error between the computational model of the experiment and the experimental temperatures is minimized. This sum of squares of temperature error is given by

$$
S=\sum_{i=1}^{N_{s}} \sum_{j=1}^{N_{r}}\left(Y_{i j}-T_{i j}\right)^{2} .
$$

Using Newton's method the iterative solution to this minimization problem for a single parameter can be written as i

2. In reality the model could be $1-D$, but it was implemented in a 2-D code. 


$$
\Delta k^{v+1}=\frac{\sum_{i=1}^{N_{s}} \sum_{j=1}^{N_{i}}\left(Y_{i j}-T_{i j}^{\nu}\right) \frac{\partial T_{i j}}{\partial k}}{\sum_{i=1}^{N_{s}} \sum_{j=1}^{N_{i}}\left(\frac{\partial T_{i j}}{\partial k}\right)^{2}},
$$

where $\Delta k^{v+1}$ is the correction to the estimated thermal conductivity for iteration $v+1$. We utilized software designed specifically to compute both temperature and sensitivity coefficient fields. Details of this methodology can be found in Blackwell et al. (1999). This software utilizes a Control Volume Finite Element Method to discretize the energy equation and also the derived sensitivity partial differential equations. It is felt that the sensitivity equation method is more accurate than finite difference determination of sensitivity coefficients and requires less user intervention to determine appropriate finite difference step sizes. The communication between the analysis software that evaluates the temperature and sensitivity coefficient fields and the software that computes the next conductivity guess is through external files and UNIX ${ }^{\mathrm{TM}}$ shell scripts. An outline of this process is presented in Blackwell and Eldred (1997) and Dowding and Blackwell (1998).

The computational model consisted of the walls of the stainless steel cylinder. The end boundary conditions were the experimentally measured temperatures at Stations 1 and 14; these temperatures were assumed uniform at these cross sections and to be errorless. The assumed boundary conditions on the side walls of the cylinder were adiabatic. The adiabatic assumption was confirmed by examining the steady state temperature profile for a run. Theoretical arguments suggest a linear temperature profile and the experimental results were consistent with this. The estimated thermal conductivity was $14.57 \mathrm{~W} / \mathrm{m}-\mathrm{K}$; this result compares favorably with other measurements given in Table 1; the other measurements were

Table 1: Comparison of thermal conductivity of 304 Stainless Steel with other results. Units are $\mathrm{W} / \mathrm{m}-\mathrm{K}$ and are valid for $31^{\circ} \mathrm{C}$; uncertainty bound is $\pm 2 \sigma$

\begin{tabular}{|c|c|c|}
\hline This Work & Taylor et al. (1997) & Incropera and De Witt (1990) \\
\hline \hline $14.57 \pm 0.6$ & $14.65 \pm 1.5$ & 14.97 \\
\hline
\end{tabular}

linearly interpolated at a temperature of $31^{\circ} \mathrm{C}$, the average temperature of Run 032100. The uncertainty statement contained in Taylor et al. (1997) stated that the conductivity was accurate to $\pm 5 \%$. It is not known if this uncertainty bound was $1 \sigma$ or $2 \sigma$; it was assumed to be $1 \sigma$. The details of the uncertainty analysis for this work are presented in a subsequent section.

Beck and Arnold (1977) emphasize the importance of "sequential" estimation of parameters. Sequential estimation considers the effect of sequentially adding data from one more measurement time on the estimated parameter. If one assumes that the sensitivity coefficients can be linearized about the converged parameter value, then Eq. (2) can be utilized to study this effect. If $N_{t}$ in Eq. (2) is sequentially increased by one up to its maximum value, then the sequentially estimated conductivity can be evaluated and these results are shown in Fig. 8. There is a

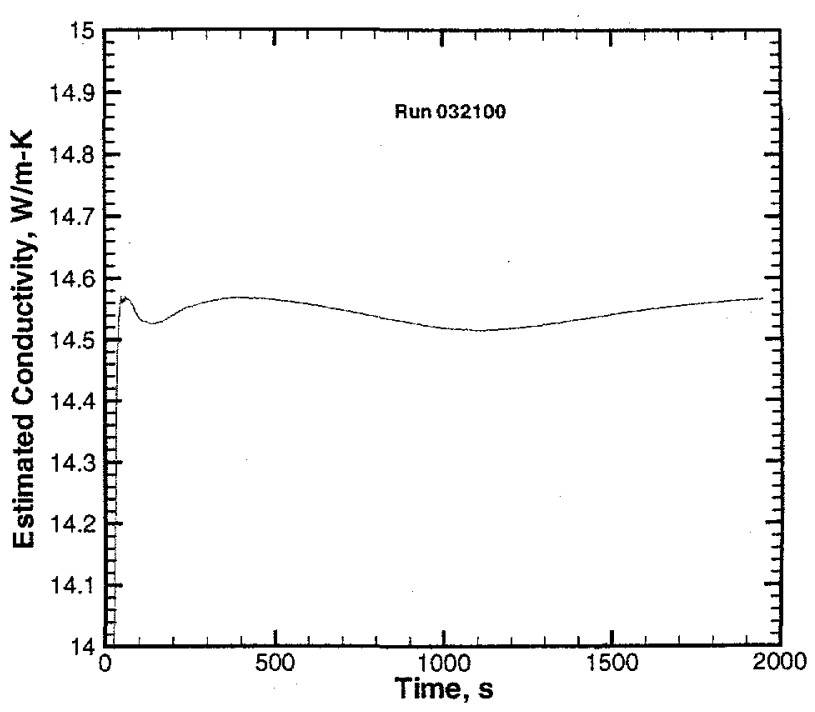

Figure 8. Sequentially estimated conductivity.

slight variation because of the addition of the cool down data. The sequential results suggest that adding more information will not substantially alter the final estimated conductivity value.

Earlier work from this same apparatus was presented in Blackwell et al. (2000) and gave an estimated conductivity of $14.34 \mathrm{~W} / \mathrm{m}-\mathrm{K}$. However, refinements of the apparatus have been made, and we feel the current data is more reliable. These refinements eliminated a minor heat loss that was not accounted for in the parameter estimation model. Also, the end-to-end symmetry was also significantly improved.

In parameter estimation it is important to study the residuals to aid in evaluating the fitted model. Fig. 9 presents the temperature residuals for the model using the estimated value of thermal conductivity. Ideally these residuals should be randomly distributed about zero. This is obviously not the case in Fig. 9; the residuals for different stations are clearly separated, and there are distinct time trends for each station's residuals including a pattern reversal at the point at which rapid cooling is initiated. In part these patterns reflect the standard deviation patterns in Fig. 6 . The residuals are, however, quite small for most practical purposes. The maximum absolute residual is about $0.07^{\circ} \mathrm{C}$, and the RMS of all the residuals is about $0.03^{\circ} \mathrm{C}$. These values should not be taken as prediction errors pertaining to temperatures in a repeat of this experiment or in another similar experiment because the conductivity used in the model calculations was obtained from the same data from which the residuals are calculated.

The mean square error in temperature was estimated from the 


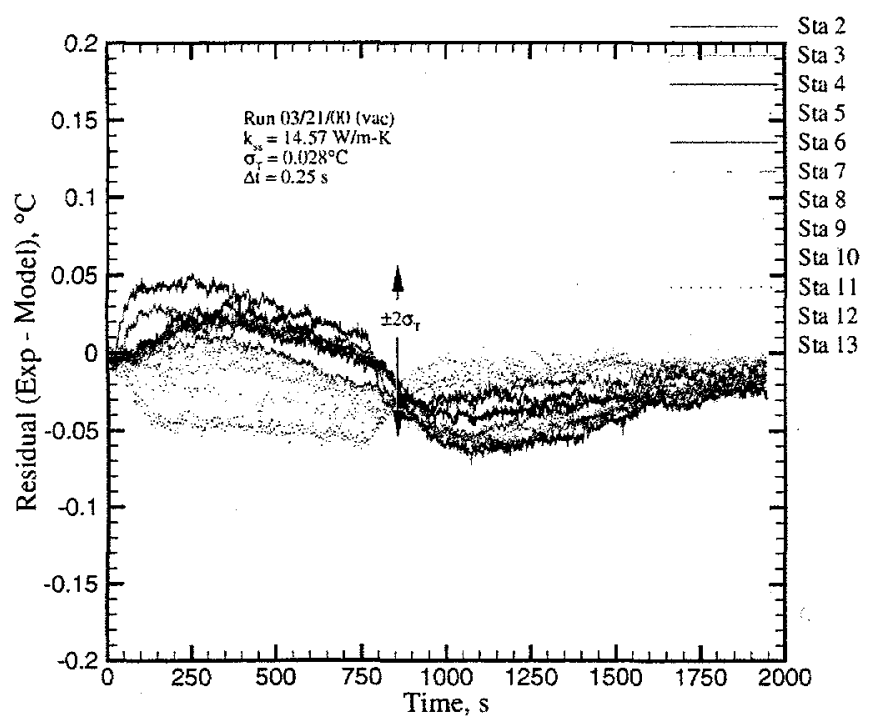

Figure 9. Temperature residuals for Run 032100.

temperature residuals using

$$
\hat{\sigma}_{T}^{2}=\frac{1}{N_{s} N_{t}-1} \sum_{i=1}^{N_{s}} \sum_{j=1}^{N_{t}}\left(Y_{i j}-T_{i j}\right)^{2} .
$$

Note that Eq. (1) and Eq. (3) differ only by a constant.

In parameter estimation it is advisable to explore the sum of squares function (or mean square error) in the neighborhood of the converged point. Fig. 10 presents the residual based standard

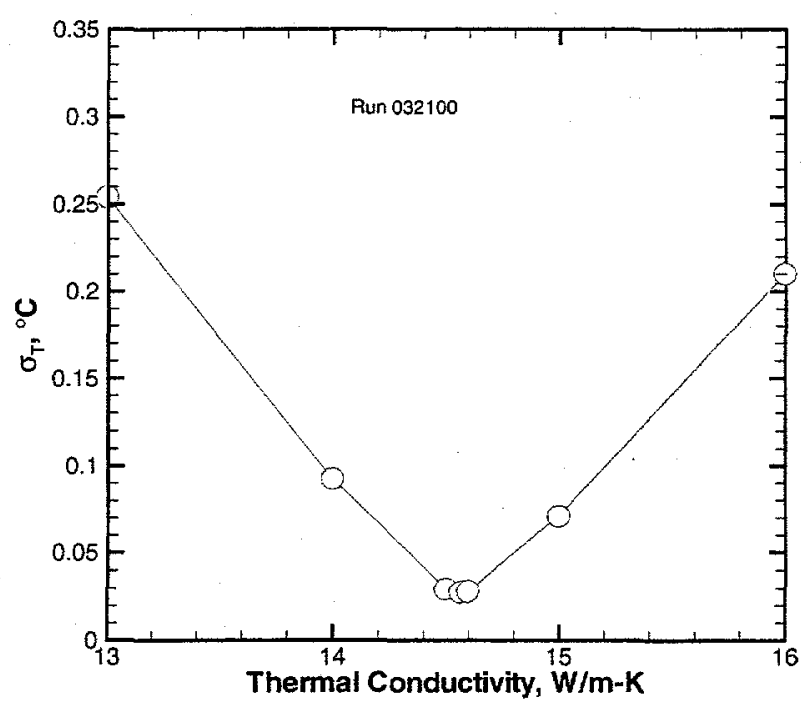

Figure 10. Estimated standard deviation in temperature vs. conductivity for Run 032100 .

deviation as a function of the assumed thermal conductivity. In close proximity to the minimum value, the space is relatively flat. When the thermal conductivity becomes significantly removed from the converged value, the standard deviation in temperature is a strong function of thermal conductivity. We believe the optimization process converged to a global minimum.

\section{EXPERIMENT DESIGN ISSUES}

The optimal design of experiments studies sensitivity coefficients in order to make decisions on quantities, such as experiment duration, sensor locations, sample rate, etc. In this instance sensitivity coefficients are defined as partial derivatives of temperature with respect to conductivity. We have found it useful to utilize scaled sensitivity coefficients. For our experiments the scaled thermal conductivity sensitivity coefficient of interest is defined as

$$
T_{k}(\grave{x}, t, k)=k \frac{\partial T}{\partial k} .
$$

Note that the scaled sensitivity coefficient is a field variable just like temperature, and it has the units of temperature. The scaling of the sensitivity coefficient is important in that it allows sensitivity coefficients to be directly compared to a characteristic temperature rise of the experiment. For this experiment the characteristic temperature rise is the rise from its initial value. For constant thermal diffusivity one can demonstrate that

$$
k \frac{\partial T}{\partial k}=\alpha \frac{\partial T}{\partial \alpha}
$$

Consequently, the two sensitivity coefficients can be used interchangeably.

The scaled thermal conductivity sensitivity coefficients for Run 032100, Stations 1-7 are presented in Fig. 11. Since the boundary conditions for Stations 1 and 14 are quite similar, the sensitivity coefficients for Stations $8-14$ will be similar to those for Stations 1-7. First, note that the sensitivity coefficient for Station 1 is zero everywhere since it is a specified temperature boundary condition. Once the experiment time exceeds about $150 \mathrm{~s}$, there is a clear trend of increasing sensitivity to thermal conductivity as you move away from the specified temperature boundary condition. Those sensors closest to the axial midplane are more sensitive to the thermal conductivity. The time at which each thermocouple has a maximum sensitivity coefficient varies. At approximately 750 s the upper copper block is suddenly cooled; this time is greater than the time at which the sensitivity coefficients are a maximum.

Note the magnitude of the scaled sensitivity coefficients rellative to the maximum temperature rise of approximately $13^{\circ} \mathrm{C}$. The sensitivity coefficients for sensors near the axial midplane are quite significant relative to the maximum temperature rise.

The duration of the heating phase (750s) of Run 032100 (Fig. 


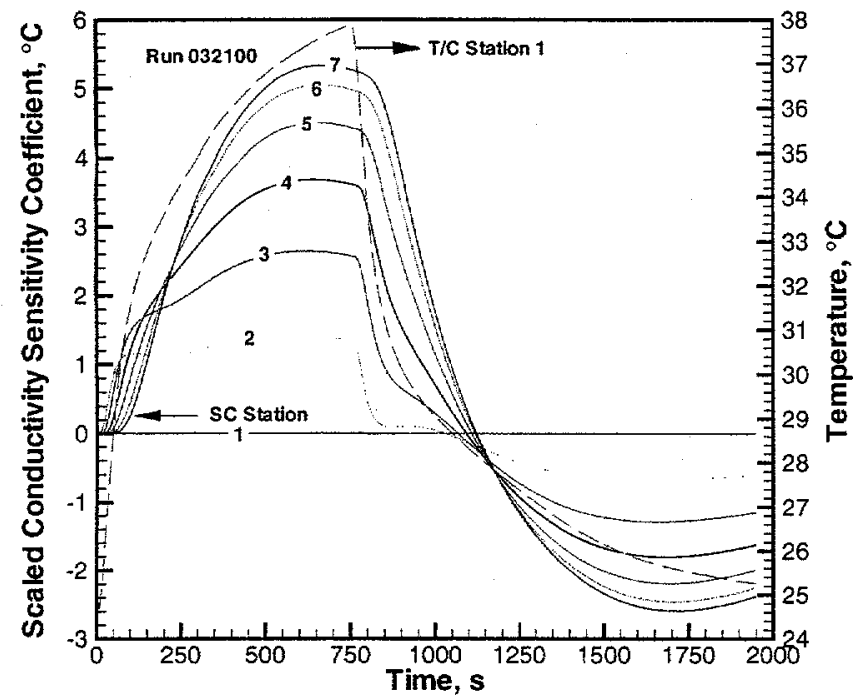

Figure 11. Scaled thermal conductivity sensitivity coefficients (left scale) and Station 1 temperature history (right scale).

3) was chosen based on the previous results of Blackwell et al. (2000). The D-optimality condition discussed in Beck and Arnold (1977) was used to guide this process. This condition involves maximizing the determinant of the matrix

$$
\Delta=\operatorname{Det}\left(X^{T} X\right),
$$

where $X$ is the $N_{s} \cdot N_{t}$ by $N_{p}$ sensitivity matrix; the number of sensors is $N_{s}$, the number of times is $N_{t}$, and the number of parameters is $N_{p}$. In this particular application, we are only estimating a single parameter, thermal conductivity of the stainless steel; hence, $N_{p}=1$. There are 14 sensors on the stainless steel cylinder, but since Stations 1 and 14 will be used as boundary conditions, there are only 12 axial stations contributing parameter estimation information; the sensitivity coefficients are zero at the specified temperature boundary conditions. The number of measurement times will be treated as variable through the relationship

$$
t=N_{t} \Delta t
$$

where $t$ is the experiment duration and $\Delta t$ is the data sample rate; for all the results presented here, $\Delta t=1.5 \mathrm{~s}$. For this simple case of a single parameter, the optimality conditions reduces to

$$
\Delta=\sum_{i=1}^{N_{s}} \sum_{j=1}^{N_{i}}\left(\left.\frac{\partial T}{\partial k}\right|_{i, j}\right)^{2}
$$

One could increase $\Delta$ by adding sensors or taking more measurements, as long as $\partial T / \partial k$ is nonzero. Furthermore, because $\partial T / \partial k$ is typically related to the temperature range, a larger temperature range causes $\Delta$ to increase. To eliminate these dependencies, a normalized version of the optimality condition was used in this study and is defined to be

$$
\Delta^{+}=\frac{1}{N_{s} N_{t}\left(T_{\max }-T_{\min }\right)^{2}} \sum_{i=1}^{N_{s}} \sum_{j=1}^{N_{t}}\left(\left.k \frac{\partial T}{\partial k}\right|_{i, j}\right)^{2},
$$

where $\left(T_{\max }, T_{\min }\right)$ is the (maximum, minimum) temperature over time and sensor location. The quantity $\Delta^{+}$can be viewed as an information content per data sample, and we want to choose the heating and cooling durations that maximize this quantity. The time dependence of $\Delta^{+}$comes through the implicit dependence of $N_{t}$ on time, Eq. (7).

Note the presence of the scaled thermal conductivity sensitivity coefficients in Eq. (9). These scaled sensitivity coefficients are precisely those shown in Fig. 11.

The D-optimality condition defined in Eq. (9) for Run 032100 is presented in Fig. 12. The heating duration for this experiment

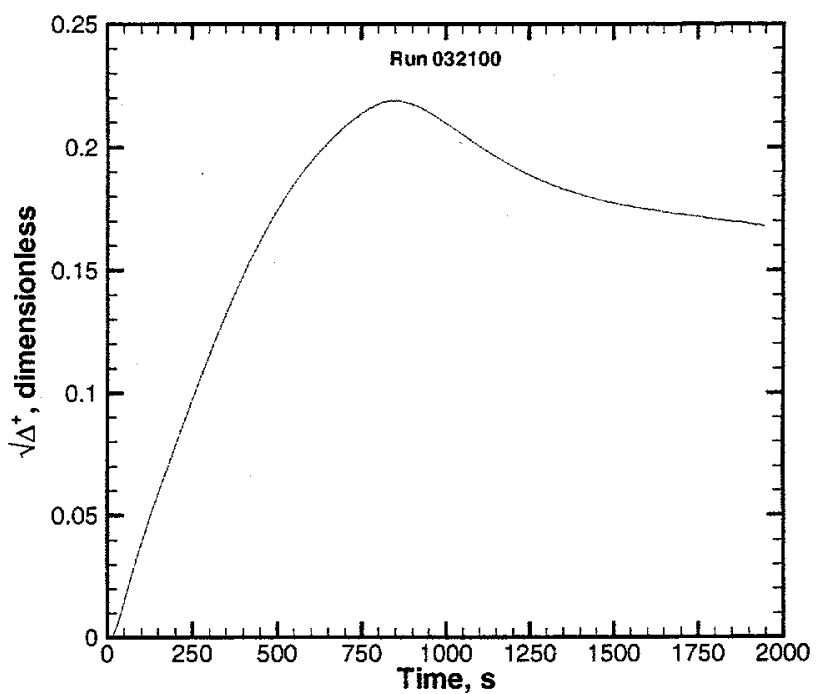

Figure 12. D-optimality condition as a function of time for Run 032100.

was $750 \mathrm{~s}$; beginning at this time cooling fluid was circulated through the top and bottom copper blocks. The cooling duration can be treated as a variable to be selected based on the time at

which $\Delta^{+}$in Fig. 12 is a maximum; this time is 850.5 s. Even though the experiment was run for $1950 \mathrm{~s}$, the majority of the information about thermal conductivity is contained in the first $850.5 \mathrm{~s}$ of the experiment. The data was reduced using a run duration of 1950 s. 


\section{UNCERTAINTY IN ESTIMATED THERMAL DIFFUSIV- ITY}

Since time-dependent temperature data is used in the parameter estimation process, the computational model requires both thermal conductivity and volumetric heat capacity. The use of temperature boundary conditions to drive the model precluded the possibility of estimating both conductivity and volumetric heat capacity from the temperature measurements presented here. The conductivity estimation process was performed by assuming a value for the volumetric heat capacity and then minimizing the least-squares error in Eq. (1). If the volumetric heat capacity is arbitrarily changed, then the resulting conductivity estimate will also change such that the ratio (thermal diffusivity) is a constant. Consequently, the parameter estimation process really estimates the thermal diffusivity. The density and heat capacity values used in the data reduction process were $\rho=7916 \pm 18 \mathrm{Kg} / \mathrm{m}^{3}$ (this work, $1 \sigma$ ) and $c=487.9 \pm 10 \mathrm{~J} / \mathrm{Kg}-\mathrm{K}$ (Taylor et al., 1997; given as $2 \%$ accuracy, assumed to be $1 \sigma$ ).

Beck and Arnold (1977) indicate that for additive, uncorrelated errors with zero mean, the standard deviation in the estimated thermal diffusivity is related to the standard deviation in the temperature measurements through

$$
\hat{\sigma}_{\alpha}^{2}=\frac{1}{\Delta} \hat{\sigma}_{T}^{2}, \text { or } \hat{\sigma}_{\alpha}=\frac{1}{\sqrt{\Delta}} \hat{\sigma}_{T} .
$$

This relationship points out why maximizing $\Delta$ is important in terms of minimizing the errors in the estimated conductivity. The dimensional and dimensionless $\Delta$ are related through Eq. (8), and Eq. (9) and can be written as

$$
\Delta=\frac{N_{s} N_{t} \Delta T^{2}}{\alpha^{2}} \Delta^{+} .
$$

The variance and standard deviation in the estimated thermal diffusivity can be written as

$$
\left(\frac{\hat{\sigma}_{\alpha}}{\alpha}\right)^{2}=\frac{1}{N_{s} N_{t} \Delta^{+}}\left(\frac{\hat{\sigma}_{T}}{\Delta T}\right)^{2} \text {, or } \frac{\hat{\sigma}_{\alpha}}{\alpha}=\frac{1}{\sqrt{N_{s} N_{t} \Delta^{+}}} \frac{\hat{\sigma}_{T}}{\Delta T} \text {. }
$$

The issue we are faced with now is how to estimate $\hat{\sigma}_{T}$. This will be explored in more detail in the following section.

\section{SUMMARY OF ESTIMATES OF STANDARD DEVIA- TION IN TEMPERATURE MEASUREMENTS}

Residuals for Run 032100 are presented in Fig. 9, and the standard deviation in the temperature was estimated from Eq. (3); it was found to be $0.0297^{\circ} \mathrm{C}$. This estimate of $\hat{\sigma}_{T}$ will be termed "residual based."

From the results in Fig. 6, the maximum circumferential standard deviation is approximately $0.16^{\circ} \mathrm{C}$ for Stations 2 and 14. Since we averaged the four thermocouples at each axial station, the estimated standard deviation must be reduced by $\sqrt{4}$. Using the maximum axial temperature gradient computed from the experimental data, the thermocouple position uncertainty of $\pm 0.44 \mathrm{~mm}( \pm 0.017 \mathrm{in})$ translates to an estimated temperature error of $\pm 0.13^{\circ} \mathrm{C}$

In summary, the three temperature uncertainty estimates vary by half an order of magnitude and lie in the approximate range $0.03<\hat{\sigma}_{T}<0.13^{\circ} \mathrm{C}$. These results are summarized in Table 2 .

Table 2: Estimated standard deviation in temperature using various methods

\begin{tabular}{|c|c|}
\hline method & $\pm 1 \hat{\sigma}_{T},{ }^{\circ} \mathrm{C}$ \\
\hline \hline residual & 0.03 \\
\hline maximum circumferential average & 0.08 \\
\hline $\mathrm{t} / \mathrm{c}$ position error of $0.44 \mathrm{~mm}(0.017 \mathrm{in})$ & 0.13 \\
\hline
\end{tabular}

\section{UNCERTAINTY IN ESTIMATED THERMAL CONDUC- TIVITY}

To account for the uncertainty in the estimated thermal conductivity, we focus on the relationship between the thermal conductivity, thermal diffusivity, density, and specific heat

$$
k=\alpha \rho c .
$$

The uncertainty in the conductivity is related to the uncertainty in the other parameters through

$$
\left(\frac{\hat{\sigma}_{k}}{k}\right)^{2}=\left(\frac{\hat{\sigma}_{\alpha}}{\alpha}\right)^{2}+\left(\frac{\hat{\sigma}_{\rho}}{\rho}\right)^{2}+\left(\frac{\hat{\sigma}_{c}}{c}\right)^{2} \text {. }
$$

The estimated uncertainty in the thermal diffusivity can be obtained from Eq. (12), yielding

$$
\left(\frac{\hat{\sigma}_{k}}{k}\right)^{2}=\frac{1}{N_{s} N_{t} \Delta^{+}}\left(\frac{\hat{\sigma}_{T}}{\Delta T}\right)^{2}+\left(\frac{\hat{\sigma}_{\rho}}{\rho}\right)^{2}+\left(\frac{\hat{\sigma}_{c}}{c}\right)^{2} .
$$

Utilizing the most pessimistic estimate in Table 2 for the standard deviation in temperature $\left(0.13^{\circ} \mathrm{C}\right)$ and the other parameters for this experiment, Eq. (15) yields

$$
\left(\frac{\hat{\sigma}_{k}}{k}\right)^{2}=1.3 \times 10^{-9}+5.2 \times 10^{-6}+4.0 \times 10^{-4} \text {. }
$$


It has been assumed that the confidence level of the various uncertainties in Eq. (15) are the same. Clearly the uncertainty in the specific heat dominates the uncertainty in the thermal conductivity. The error in temperature measurement is not a consideration because the temperature measurements were assumed to be independent. A refined error analysis that assumed correlated errors has been performed by one of us (RGE), and while increasing the first term in Eq. (16) by a factor of roughly 300 , the temperature measurement error is still an order of magnitude smaller than the next largest term. The final estimated thermal conductivity and associated uncertainty is

$$
k=14.57 \pm 0.6 \mathrm{~W} / \mathrm{m}-\mathrm{K}( \pm 2 \sigma \text { bounds }) .
$$

Any significant reductions in uncertainty in thermal conductivity estimate must be accompanied by a reduction in the $(2 \%)$ uncertainty in the specific heat.

\section{SUMMARY}

The experimental configuration for estimating thermal conductivity of 304 stainless steel was axial heat conduction in the walls of a hollow cylinder. Optimum experiment design issues were discussed. Sensitivity coefficients and the Doptimality condition were computed. The estimated thermal conductivity at $31^{\circ} \mathrm{C}$ was $14.57 \mathrm{~W} / \mathrm{m}-\mathrm{K}$, which compares favorably with other estimates. An uncertainty analysis on the temperature measurements was performed using three different methods with the results given in Table 2 . The uncertainty in the specific heat is the dominant factor in the conductivity uncertainty. In order to further reduce the uncertainty in the thermal conductivity, the uncertainty in the estimated heat capacity will have to be reduced.

\section{ACKNOWLEDGMENTS}

The authors acknowledge the support of Pat Drozda and Chuck Hanks of Sandia for the fabrication of the apparatus, writing of the data acquisition software, and conduct of the experiments.

\section{REFERENCES}

Beck, J. V. and Arnold, K. J., 1977, Parameter Estimation in Engineering and Science, Wiley, New York.

Blackwell, B. F. and Eldred, M. S., 1997, "Application of Reusable Interface Technology for Thermal Parameter Estimation, in G. S. Dulikravich and K. A. Woodbury (eds), Proceedings 32nd National Heat Transfer Conference, vol. 2, Inverse Problems in Heat Transfer and Fluid Flow,"ASME HTD-Vol. 340, pp. 1-8.

Blackwell, Bennie F. and Dowding, Kevin J, 1999, "Sensitivity Analysis and Uncertainty Propagation in a General Purpose Thermal Analysis Code," Proceedings of the 3rd ASME/JSME Joint Fluids Engineering Conference, July 18-23, San Francisco, CA.
Blackwell, Bennie F., Dowding, Kevin J., and Cochran, Robert J., 1999, "Development and Implementation of Sensitivity Coefficient Equations for Heat Conduction Problems," Numerical Heat Transfer, Part B, Vol 36, pp 15-32.

Blackwell, Bennie F., Gill, Walter, Dowding, Kevin J., and Voth, Thomas E., 2000, "Determination of Thermal Conductivity of 304 Stainless Steel Using Parameter Estimation Techniques," Proceedings of 34th National Heat Transfer Conference.

Coleman, Hugh W. and Steele, W. Glenn, 1999, Experimentation and Uncertainty Analysis for Engineers, 2nd Ed., Wiley, NY.

Dowdell, R. B., 1982, ASME Joumal of Fluids Engineering, Vol. 104, No. 2, p. 258.

Dowding, K. J. and Blackwell, B. F., 1998, "Joint Experimental/ Computational Techniques to Measure Thermal Properties of Solids," Measurement Science and Technology, Vol. 9, No. 6, pp. 877-887.

Incropera, Frank P. and De Witt, David P., 1990, Introduction to Heat Transfer, 2nd Edition, Wiley, NY.

Roache, Patrick J., 1998, Verification and Validation in Computational Science and Engineering, Hermosa Publishers, Albuquerque.

Stern, Fred, Wilson, Robert, Coleman, Hugh W., and Paterson, Eric G., 1999, Proceedings of the 3rd ASME/JSME Joint Fluids Engineering Conference, FED-Vol. 248, pp.1-15.

Taylor, R. E., Groot, H., and Ferrier, J., 1997, "Thermophysical Properties of SS 304," TPRL 1904, Thermophysical Properties Research Laboratory, Purdue University. 